Cahiers $d u$ MONDE RUSSE

\section{Cahiers du monde russe}

Russie - Empire russe - Union soviétique et États indépendants

$41 / 1 \mid 2000$

Varia

\title{
Le rapatriement des citoyens soviétiques à partir de la France et des zones d'occupation françaises en Allemagne et en Autriche
}

Pavel M. Polian

\section{(2) OpenEdition}

Édition électronique

URL : https://journals.openedition.org/monderusse/35

DOI : $10.4000 /$ monderusse.35

ISSN : $1777-5388$

Éditeur

Éditions de l'EHESS

\section{Édition imprimée}

Date de publication : 1 janvier 2000

Pagination : 165-190

ISBN : 2-7132-1353-3

ISSN : $1252-6576$

Référence électronique

Pavel M. Polian, "Le rapatriement des citoyens soviétiques à partir de la France et des zones d'occupation françaises en Allemagne et en Autriche », Cahiers du monde russe [En ligne], 41/1 | 2000, mis en ligne le 15 janvier 2007, consulté le 03 septembre 2022. URL : http://journals. openedition.org/ monderusse/35 ; DOl : https://doi.org/10.4000/monderusse.35 


\section{LE RAPATRIEMENT DES CITOYENS SOVIÉTIQUES DEPUIS LA FRANCE ET LES ZONES FRANÇAISES D'OCCUPATION EN ALLEMAGNE ET EN AUTRICHE*}

EN COMPARAISON AVEC LE RAPATRIEMENT DES CITOYENS SOVIÉTIQUES depuis l'Angleterre et les États-Unis et leurs zones d'occupation, celui qui eut lieu depuis la France et les zones françaises d'occupation en Allemagne et en Autriche après la guerre est un sujet de recherche trop peu exploré. Hormis quelques mentions d'ordre plutôt général trouvées çà et là dans des études anglophones et germanophones, il n'y a guère qu'une publication d'un auteur français sur laquelle on puisse attirer l'attention, la monographie de Georges Coudry intitulée Les camps soviétiques en France. Les «Russes » livrés à Staline en 19451, dans laquelle ces questions font l'objet d'une étude spécifique. Nous traitons également de ce sujet dans notre monographie intitulée Žertvy dvuh diktatur ${ }^{2}$, mais dans le contexte beaucoup plus large du rapatriement des citoyens soviétiques dans son ensemble. Dans le présent article nous examinons les problèmes relatifs uniquement au rapatriement depuis la France et ses zones d'occupation, puisant à la fois dans des sources

\footnotetext{
* Nous adressons nos vifs remerciements à nos collègues français (N. Werth et G. Coudry), autrichiens (K. Eisterer et S. Karner) et allemands (P. Fessler et K. Henks), au personnel des fonds d'archives de Moscou, Paris et Colmar pour leur aide, et plus particulièrement à la fondation de la Maison des sciences de l'homme dont le soutien nous a permis de poursuivre notre recherche dans les archives et les bibliothèques françaises en mai et juin 1998.

1. Georges Coudry, Les camps soviétiques en France. Les « Russes » livrés à Staline en 1945, Paris, Albin Michel, 1997.

2. Pavel M. Poljan, Žertvy dvuh diktatur. Ostarbajtery $i$ voennoplennye $v$ Tret'em Rejhe $i$ ih repatriacija (Victimes de deux dictatures. Les travailleurs obligatoires et les prisonniers de guerre du III ${ }^{\mathrm{e}}$ Reich et leur rapatriement), Moscou, Vaš Vybor CIRZ, 1996.
} 
d'archives déjà connues et dans celles qui ont été récemment découvertes ainsi que dans les sources imprimées.

\section{Les frontières de l'Europe d'après-guerre}

Les frontières de l'Europe d'après-guerre, qui furent si nettement définies dans le courant de l'été 1945 lors de la Conférence de Berlin (Potsdam) tenue du 17 juillet au 2 août dans les salles du Cecilienhof, étaient déjà dessinées depuis longtemps.

Ainsi, le morcellement du territoire allemand en zones alliées d'occupation avait-il déjà été prévu dans un Protocole d'accord passé entre les gouvernements américain, britannique et soviétique, et signé le 12 septembre $1944^{3}$. Les frontières entre les zones avaient été assez nettement définies, chaque zone étant occupée par les troupes d'une seule des puissances victorieuses; on avait nommé un commandant en chef pour chacune d'entre elles, et c'est seulement pour le Grand Berlin que l'on avait prévu un organe collectif interallié.

À la Conférence de Yalta en février 1945, il fut décidé que la France serait associée à l'occupation de l'Allemagne et l'on créa une Commission de démembrement de l'Allemagne dont les séances se tinrent à Londres à partir de mars 1945. La France s'était jointe aux activités de la Commission consultative européenne dès la fin de 1944. Le 5 juin fut signée à Berlin ce que l'on appelle la «Déclaration de Berlin », à savoir la déclaration commune de la capitulation de l'Allemagne et l'auto-attribution de l'autorité suprême sur l'Allemagne par les gouvernements de l'URSS, de la Grande-Bretagne, des États-Unis, et par le gouvernement provisoire de la République française. L'accord fut signé par les commandants en chef des forces armées des États alliés sur le sol allemand : le maréchal Žukov, le général Eisenhower, le maréchal Montgomery et le général de Lattre de Tassigny. Entre les quatre gouvernements fut conclu un accord sur les zones d'occupation et sur le Conseil de contrôle qui était composé des commandants en chef des forces armées des Alliés présents en Allemagne, et dont toutes les décisions devaient être adoptées à l'unanimité.

Le lendemain (6 juin), fut formée l'Administration militaire soviétique chargée de la direction de la zone d'occupation soviétique (plus communément connue sous son autre nom, Administration militaire soviétique en Allemagne, ou SVAG

3. Lors de l'adoption par la Commission consultative européenne de l' « Accord sur les procédures de contrôle en Allemagne » du 14 novembre 1944 furent introduits des amendements précisant le tracé de la frontière entre les zones anglaise et américaine, après quoi le protocole fut expédié aux gouvernements des trois pays qui le ratifièrent tous sans le modifier, la GrandeBretagne le 5 décembre 1944, les États-Unis le 2 février 1945, et l’URSS le 6 février 1945 (pour un compte rendu détaillé, cf. G. P. Kynin, « Germanskij vopros vo vzaimootnošenijah SSSR, SŸA i Velikobritanii, 1944-1945 gg. » (La question allemande dans les relations entre l'URSS, les USA et la Grande-Bretagne, 1944-1945), Novaja i novejšaja istorija, 4, 1995, pp. 105-132). 
(Sovetskaja Voennaja Administracija v Germanii) ${ }^{4}$. Le maréchal G. K. Žukov fut nommé administrateur général de la $\mathrm{SVAG}^{5}$, et comme adjoints furent nommés le général d'armée V. D. Sokolovskij (premier adjoint), le commissaire de la défense nationale de deuxième rang I. A. Serov (chargé de l'administration civile) et M. Z. Saburov6; le colonel général V. V. Kurasov fut nommé chef d'état-major de juin à octobre 1945, ensuite il céda la place au lieutenant général M. I. Dratvin7 ${ }^{7}$ Dans un premier temps (jusqu'en août 1945), A. Ja. Vyšinskij fut le conseiller politique de l'administrateur général de la SVAG; ce fut ensuite le tour d'A. S. Sobelev, et, du 31 mai 1946 jusqu'à la formation de la RDA, de V.S. Semenov. Par ordre de Žukov, les dirigeants des administrations militaires soviétiques dans les provinces et territoires fédérés ainsi que leurs adjoints aux affaires civiles furent nommés au fur et à mesure à partir du 9 juillet 1945.

Faut-il rappeler que les Alliés avaient eux aussi formé des organes similaires dans leurs zones d'occupation sous mandat en Allemagne et en Autriche. Les tout premiers chefs suprêmes des armées furent le feld-maréchal Montgomery dans la zone britannique, le général Dwight Eisenhower dans la zone américaine et le général de Lattre de Tassigny dans la zone française. Dès le 15 juin 1945, de Tassigny fut remplacé par le général Marie-Pierre Kœnig8 dont le quartier général était installé à Baden-Baden (avec le temps son poste revint au gouverneur général François-Poncet qui resta en RDA comme ambassadeur après la disparition des zones d'occupation). Eisenhower fut remplacé par le général Lucius Clay, et Montgomery par le maréchal C. Douglas et, en mars 1946, par le général B. Robertson.

Parallèlement, les Alliés (ou plus précisément la Commission consultative européenne de Londres) s'occupaient aussi de la question autrichienne. Celle-ci présentait, il faut le dire, une différence majeure : alors que l'Allemagne représentait à leurs yeux un État vaincu, l'Autriche, elle, était un État libéré. Le 4 juillet 1945 fut

4. Décret du SNK (Sovet Narodnyh Komissarov - Conseil des commissaires du peuple) de l'URSS n 1326/301ss. Pour une description détaillée de la SVAG, cf. M. I. Semirjaga, Kak my upravljali Germaniej. Politika i žizn' (Comment nous gouvernâmes l'Allemagne. Politique et vie quotidienne), Moscou, Rospen, 1995; SBZ-Handbuch. Staatliche Verwaltungen, Parteien, gesellschaftliche Organisationen und ihre Führungskräfte in der sowjetischen Besatzungszone Deutschlands 1945-1949, ed. par M. Broszat et H. Weber, 2e éd., Munich, R. Oldenbourg Verlag, 1993.

5. Il restait en même temps commandant en chef du groupe des troupes soviétiques en Allemagne. Il demeura à son poste jusqu'en mars 1946, date à laquelle il fut remplacé par le maréchal V. D. Sokolovskij; le général d'armée V. V. Čujkov fut commandant en chef de mars à octobre 1949.

6. M.Z. Saburov fut bientôt remplacé par K. I. Koval', commissaire du peuple adjoint à l'Industrie lourde de l'URSS pendant la guerre et dirigeant de la partie soviétique du Directorat économique de la Commission de contrôle soviétique (SKK).

7. Jusqu'en mai 1947, date à laquelle M. I. Dratvin devint l'adjoint de l'administrateur général. Le lieutenant général G. S. Luk'jančenko lui succéda au poste de chef d'état-major ( $S B Z$ Handbuch..., op. cit., p. 50).

8. De 1945 à 1947, c'est le socialiste réformateur Émile Laffon qui le remplaça aux affaires civiles. 
adopté à Londres un projet d'accord sur un appareil de contrôle en Autriche et le traité correspondant fut signé 9 . Le texte d'accord définitif sur les zones d'occupation en Autriche et la gestion gouvernementale de Vienne fut adopté le 9 juillet ${ }^{10}$. Le 4 juillet fut formé l'organe de gouvernement militaire en Autriche Orientale (la partie soviétique de la Commission alliée en Autriche) ${ }^{11}$. À sa tête se trouvait le maréchal I. S. Konev, commandant du premier groupe d'armées ukrainien qui fut nommé commissaire militaire (plus tard haut-commissaire) de l'URSS en Autriche en septembre 1945 et eut comme adjoint le colonel général A. S. Želtov ${ }^{12}$.

Du côté américain, le poste correspondant fut occupé par le général Mark W. Clark de septembre 1945 à décembre 1946, ensuite par le lieutenant général Jeffrey Case de janvier 1947 à octobre 1950. Du côté britannique, ce fut, de septembre 1945 à avril 1946 le lieutenent général Sir Richard MacCrickey, et de mai 1946 à septembre 1947, le lieutenant général Sir James Steel13. Du côté français, ce fut le général d'armées Marie-Émile Batou (Béthouart) de septembre 1945 à septembre 1950. Les quartiers généraux du commandement militaire des Alliés étaient situés ainsi : l'URSS à Baden (où se trouvait également le commandement des forces armées stationnées en Tchécoslovaquie et en Hongrie), les États-Unis à Salzbourg, la France à Innsbruck, et la Grande-Bretagne à Klagenfurt.

\section{Les accords sur le rapatriement}

Le rapatriement des personnes déplacées, dont des citoyens soviétiques, français, anglais et américains, incombait aux organes qui s'étaient mis en place dans les zones d'occupation. Le fondement juridique du rapatriement forcé des citoyens soviétiques fut établi par une série de traités bilatéraux conclus entre les Alliés occidentaux et l'URSS.

Ce rapatriement forcé fut définitivement mis en place par des négociations et accords signés les 10 et 11 février 1945 à Yalta. Le 11 février, Vjačeslav Molotov et Anthony Eden signèrent, sur mandat de leur gouvernement, un accord anglo-

9. Les signataires étaient l'ambassadeur des États-Unis J. G. Winant, P. Campbell pour la Grande-Bretagne, l'ambassadeur de France R. Massigli et l'ambassadeur de l'URSS, F. Gusev. Un peu moins d'un an plus tard, le 28 juin 1946, fut signé à Vienne un deuxième traité sur l'appareil de contrôle en Autriche. Voir ces textes dans M. Rauchensteiner, Der Sonderfall. Die Besatzungszeit in Österreich 1945 bis 1955, Graz, Styria, 1995, pp. 339-342, 344-349 (Reprint).

10. Ibid., pp. 342-343.

11. Décret du SNK de l’URSS n $1553-355$ s du 4 juillet 1945.

12. Želtov tint ce poste jusqu'en juillet 1950, alors que Konev fut rappelé en avril 1946 et remplacé en mai par le colonel général V. V. Kurasov (qui fut lui-même remplacé trois ans plus tard par le lieutenant général V.P. Sviridov). E. D. Kisilev fut conseiller politique pendant toute cette période (pour plus de détails, cf. M. Rauchensteiner, op. cit., pp. 172, 351).

13. Les Britanniques, rendus inquiets par la «menace yougoslave » et les problèmes relatifs à la Carinthie, Trieste et la Vénétie Julienne, gardèrent sous les armes de grandes unités d'anciens prisonniers de guerre allemands, dont le corps d'armée du colonnel Rogožin. Pour plus de détails, cf. M. Rauchensteiner, op. cit., pp. 149-153, 351-352. 
soviétique ; et le lieutenant général A. A. Gryzlov et le major général D. R. Dean, un accord américano-soviétique ${ }^{14}$. Molotov et Eden signèrent en outre plusieurs documents, dont notamment un accord additionnel sur le statut des Russes séjournant dans des camps sur le territoire britannique ${ }^{15}$ selon lequel les citoyens soviétiques libérés par les Alliés devaient impérativement « se tenir à l'écart des prisonniers de guerre allemands », après quoi ils «seront rassemblés en des lieux spécifiques et des camps où se présenteront sans attendre les représentants soviétiques affectés au rapatriement ». L'article 2 mérite que l'on s'y arrête : « À des fins de gestion et de discipline internes, ces citoyens soviétiques seront organisés en unités et groupes soumis aux lois soviétiques ». Ainsi les Soviétiques s'octroyaientils la gestion des camps; ils confièrent aux Anglais le financement de toutes les opérations ainsi que le transport des Soviétiques jusqu'aux postes intermédiaires ou même jusqu'en URSS ${ }^{16}$. Les officiers soviétiques obtinrent bientôt des droits similaires auprès des Américains.

Un peu plus tard, le 13 mars et le 29 juin 1945, l'URSS conclut des accords similaires à ceux de Yalta avec la Belgique ${ }^{17}$ et la France. Le dernier « Accord sur la détention et le rapatriement des citoyens soviétiques et français se trouvant respectivement sous le contrôle des autorités françaises et soviétiques » fut signé à

14. Intitulés exacts des accords : «Accord relatif aux prisonniers de guerre et personnes libérées par les troupes sous commandement soviétique et les troupes sous commandement britannique [ou : sous commandement américain] ». Leur contenu était strictement identique. Cf. Krymskaja konferencija rukovoditelei treh sojuznyh deržav - SSSR, S $\ddot{Y}_{A}$ i Velikobritanii, 411 fevralja $1945 \mathrm{~g}$. : Sbornik dokumentov (La Conférence de Yalta entre les dirigeants de trois puissances, 1'URSS, les États-Unis et la Grande-Bretagne, 4-11 février 1945. Recueil de documents), Moscou, Politizdat, 1984, 4, pp. 264-267, 275-279, dans la série Sovetskij Sojuz na meždunarodnyh konferencijah perioda Velikoj Otečestvennoj vojny 1941-1945 gg. (L'Union Soviétique aux conférences internationales au moment de la Grande Guerre patriotique, 1941 1945). Gardés secrets, les textes des accords ne furent publiés pour la première fois qu'en 1955 aux États-Unis dans The Conferences at Malta and Yalta, Washington, 1955, pp. 985-987 dans la série Foreign relations of the United States et en URSS dans Sbornik dejstvujuščih dogovorov, soglašenij i konvencij, zaključennyh SSSR s inostrannymi gosudarstvami. Dejstvujuščie dogovory, soglašenija i konvencii, vstupivšie v silu meždu 22 ijunja 1941 i 2 sentjabrja 1949 (Recueil de traités, d'accords et de conventions en vigueur signés entre l'URSS et les États étrangers. Traités, accords et conventions entrés en vigueur entre le 22 juin 1941 et le 2 septembre 1949), Moscou, Gospolitizdat, 1955, fasc. 11, pp. 143-157. L'édition fut tirée à 5000 exemplaires.

15. Intitulé complet : « Accord entre les gouvernements de l'Union des Républiques Socialistes Soviétiques et du Royaume-Uni de Grande-Bretagne et d'Irlande du Nord relatif aux citoyens soviétiques libérés se trouvant au Royaume-Uni ». L'accord était accompagné de deux documents annexes: 1) «De la juridiction du Royaume-Uni sur les membres des unités soviétiques » (la juridiction britannique n'était maintenue que dans les cas de crimes graves) ; 2) « Des mesures financières réglant la délivrance de prêts octroyés par le gouvernement du Royaume-Uni aux citoyens soviétiques libérés membres d'unités soviétiques, équipement et ravitaillement de ces unités ». Cf. Krymskaja konferencija..., op. cit., 1979, pp. 270-274.

16. Ibid., pp. 290-292.

17. « Accord entre l'URSS et la Belgique sur le rapatriement de leurs citoyens 'déplacés' par la guerre» (publié pour la première fois dans Sbornik dejstvujuščih dogovorov..., op. cit., pp. 141-143, doc. $\mathrm{n}^{\circ} 466$ ). Cet accord avait ceci de particulier qu'il fut conclu à trois mois d'échéance avec un droit de prolongation de trois mois si l'une ou l'autre des parties ne déclarait pas au préalable la fin de son effet. Nous ne disposons pas de données sur la durée de l'effet de cet accord. 
Moscou par Andrej Vyšinskij, adjoint du commissaire du peuple aux Affaires étrangères ${ }^{18}$, et par l'ambassadeur de France Pierre Charpentier ${ }^{19}$. Selon plusieurs légendes qui circulèrent longtemps sur l'accord franco-soviétique (basées notamment sur des rumeurs relatives à la livraison de ressortissants baltes à l'URSS par les Français), celui-ci aurait été formulé, en ce qui concerne les personnes refusant le rapatriement, en termes beaucoup plus sévères que ceux de Yalta. Mais son texte ne confirme nullement ces dires ${ }^{20}$. Le traité est essentiellement identique à ceux de Yalta avec quelques exceptions : l'article 8 en est supprimé (pour des raisons évidentes) ${ }^{21}$ ainsi que quelques anachronismes tels que «protection assurée contre les bombardements », etc.

Le caractère distinctif fondamental de l'Accord franco-soviétique est la présence d'un protocole en deux points :

«1. Tous [souligné par l'auteur] les citoyens soviétiques et français sont soumis au rapatriement, y compris ceux qui font l'objet de poursuites pour des crimes commis dans leur pays ainsi que sur le territoire de l'autre pays signataire.

2. L'Accord cité ci-dessus sur la détention et le rapatriement des citoyens soviétiques et français s'applique également aux ressortissants des possessions et protectorats français. »

Le premier point nécessite des éclaicissements. Vers la fin de la guerre, il s'était accumulé en France un nombre considérable de citoyens soviétiques : on cite par exemple le chiffre de 94000 personnes pour l'automne de $1944^{22}$. C'étaient soit des militaires de la Wehrmacht (certaines unités russo-germaniques sur le front occidental ont laissé derrière elles le terrible souvenir de leur cruauté envers la paisible population française), ou des travailleurs obligatoires placés en Alsace, en Lorraine ou dans d'autres territoires français; il y avait un nombre non négligeable de prisonniers civils et de guerre qui avaient pu s'enfuir et rejoindre le maquis

18. Andrej Januar'evič Vyšinskij (1883-1954), juriste et ambassadeur extraordinaire et plénipotentiaire, docteur en droit, devint membre de l'Académie des sciences en 1939. Il fut le premier adjoint du commissaire du peuple aux Affaires étrangères de l'URSS de 1946 à 1949, ministre des Affaires étrangères de l'URSS de 1949 à 1953, premier adjoint du ministre des Affaires étrangères de l'URSS et représentant permanent de l'URSS à l'ONU de 1953 à 1954.

19. L'accord était ratifié le jour-même par le décret du SNK de l'URSS nº 1497-341s.

20. L'élaboration de l'accord dura environ trois mois pendant lesquels furent rapatriés vers l'URSS et la France environ 15000 hommes sur 132000 (cf. Sbornik dejstvujuščih dogovorov..., op. cit., pp. 158-161, doc. $\mathrm{n}^{\circ} 470$ ). Pour le projet d'accord et de protocole (en date du 26 juin 1945), cf. Gosudarstvennyj Arhiv Rossijskoj Federacii (GARF), 9526/4a/2/19-22. Il fut publié en France pour la première fois en 1948 mais sans le texte du protocole annexe : La Documentation française, Paris, Notes et études documentaires, 801, 1948.

21. Nous en citerons néanmoins le texte : «Les parties signataires du traité s'engagent à mettre en application le plus effectivement possible les dispositions du présent Accord exposées cidessus, tout en admettant certaines restrictions provisoires, seulement en ce qui concerne des questions de détail qui de temps en temps seront occasionnées par la situation stratégique ou les conditions de ravitaillement et de transport sur les divers théâtres d'opération ».

22. Cf. K. Eisterer, « Französische Besatzungspolitik. Tirol und Vorarlberg 1945-1946 », Innsbrucker Forschungen zur Zeitgeschichte, 9, 1992, p. 242. 
(certains ont même combattu dans les rangs de l'armée régulière française du général de Gaulle). Contrairement aux Anglais et aux Américains, les Français étaient particulièrement liés à l'URSS par le fait qu'environ 13000 ressortissants d'Alsace et de Lorraine, mobilisés de force par la Wehrmacht, avaient fourni de nouvelles recrues au Reich; de plus, un bon nombre de Français avaient, de gré ou de force, servi dans des corps de troupe auxiliaires allemands sur le front oriental. Beaucoup d'entre eux étaient naturellement tombés aux mains des Soviétiques avec la grande masse des prisonniers de guerre allemands. Cela rendait la question d'un rapatriement total et réciproque des citoyens soviétiques et français tout aussi impérative pour la France que pour l'URSS ${ }^{23}$.

\section{Les organes affectés au rapatriement}

L'URSS disposait d'organes d'État chargés d'appliquer les mesures relatives au rapatriement de ses citoyens bien avant la Conférence de Yalta. Par exemple, les hauts fonctionnaires affectés au rapatriement furent nommés le 4 octobre 1944 date à laquelle l'Armée rouge avançait déjà inexorablement vers les frontières allemandes ${ }^{24}$.

C'est le colonel général F. I. Golikov ${ }^{25}$ qui fut nommé représentant plénipotentiaire du SNK (plus tard du Conseil des ministres) de l'URSS pour le rapatriement des citoyens soviétiques d'Allemagne et des pays occupés. Il eut pour adjoints le colonel général I. V. Smorodinov 26 et le lieutenant général Konstantin

23. L'Ambassade de France à Moscou addressa, le 11 septembre 1945, une lettre au commissariat du peuple aux Affaires étrangères de l'URSS (NKID) qui, tout en remerciant l'URSS d'avoir renvoyé une partie des Français d'Alsace-Lorraine, exprimait de l'inquiétude sur l'état d'extrême épuisement des personnes rapatriées et demandait le rapatriement accéléré de celles qui restaient. Faisant référence au traité du 29 juin 1945, les Français demandaient qu'on autorise leurs officiers chargés du rapatriement à visiter, accompagnés d'officiers soviétiques, les camps appropriés (cf. Arhiv Vnešnej Politiki Rossijskoj Federacii - AVP RF, 0431/1/9/54/ 76-77; à la lettre est agrafée une attestation sur la progression du rapatriement des prisonniers de guerre français depuis l'URSS au 25 septembre 1945 : en tout 15139 personnes (ce chiffre est biffé et une inscription au crayon rouge indique que l'on avait communiqué un chiffre entre 12000 et 13000 personnes à Keller, le représentant plénipotentiaire français à Moscou); 5149 avaient été dirigées vers Francfort-sur-l'Oder, dont 2592 qui avaient déjà été livrées aux Alliés ; 7921 étaient en voie de sortir des camps du NKVD (ce chiffre aussi est biffé); 2069 personnes seraient dirigées vers Francfort-sur-l'Oder avant le $1^{\text {er }}$ octobre 1945. Ainsi nous savons que les nouvelles des Alsaciens et des Lorrains constituaient pratiquement le seul sujet de conversation des représentants des zones françaises d'occupation en Allemagne avec les prisonniers de guerre allemands revenant de l'URSS (communication de E. Nübling, ancien prisonnier de guerre).

24. Décret du SNK de l’URSS n 1315-392s du 4 octobre 1944.

25. Voir plus bas.

26. De janvier 1941 à août 1943, chef de l'état-major du front d'Extrême-Orient créé en juillet 1940 . 
Golubev $^{27}$, et comme aides, le major général V. M. Dragun ${ }^{28}$ et A. A. Smirnov ${ }^{29}$. La composition de ce groupe fut modifiée peu de temps après, suite au départ en mission de Dragun pour Paris (voir infra) : en firent dorénavant partie les majors généraux V. A. Revjakin, Jakov Dmitrievič Basilov et Hmara.

Le 6 octobre 1944 fut adopté un décret sur les fonctions du représentant plénipotentiaire du SNK chargé du rapatriement ${ }^{30}$. Le service correspondant fut mis en place le 23 octobre $^{31}$. Il s'intitula « Secrétariat du représentant plénipotentiaire du SNK de l'URSS chargé du rapatriement des citoyens soviétiques depuis l'Allemagne et les pays occupés $»^{32}$ et s'installa au centre même de Moscou, 7, rue Kropotkinskij. L'effectif en fut fixé à 200 personnes (dont 170 militaires). L'effectif des groupes à l'étranger ou des représentations était de 210 personnes au départ, mais il s'élargit à 523 avec le temps.

On comptait en outre 323 officiers chargés du rapatriement dans les troupes d'occupation ${ }^{33}$. L'une des sous-divisions de la SVAG devint notamment le «Bureau du rapatriement », renommé le 24 janvier 1946 «Bureau de rapatriement et de recherche des citoyens des Nations Unies auprès de l'état-major de la

27. Konstantin Dmitrievič Golubev (1896-1956), rejoignit l'Armée rouge en 1918, devint major général en 1941 et lieutenant général en 1942. Pendant la guerre, il commanda les 10e $13^{\mathrm{e}}$ et $43^{\mathrm{e}}$ corps d'armée (en juin et juillet 1941, juillet et août 1941 et d'octobre 1941 à mai 1944 respectivement) et fut mis ensuite à la disposition du quartier général. De 1944 à août 1949, il fut adjoint et premier adjoint du représentant plénipotentiaire du SNK de l'URSS chargé du rapatriement (il fut démis de cette fonction par le décret du Conseil des ministres de l'URSS n 3536 du 19 août 1945 et remplacé par le colonnel N. A. Filatov), ensuite il rejoignit le corps enseignant. Comme il est d'usage, c'est lui qui gérait la correspondance administrative courante et les négociations importantes avec les représentants de missions étrangères à Moscou. Eurent lieu notamment treize rencontres avec les généraux Petit et Keller, chefs de la légation française, dix avec le général Dean, chef de la légation américaine et deux autres rencontres avec les contre-amiraux Mayuls et Olsen, cinq avec le contre-amiral Archers, chef de la légation britannique, deux avec le général Hammel, et également avec des diplomates norvégiens, belges, yougoslaves et tchèques. Les 22 et 23 mai 1945, Golubev mena des négociations avec le major général Barker, adjoint au chef d'état-major, commandant en chef des forces expéditionnaires alliées qui se conclurent par la signature à Halle d'un important accord avec les Américains (GARF, 9526/1/1/50-51). De ce fait, le fardeau principal de la gestion du rapatriement reposait entièrement sur les épaules de Golubev plutôt que sur celles de Golikov (qui cumulait son poste au rapatriement avec la fonction, plus significative à ses yeux, semble-t-il, de chef du personnel du commissariat du peuple à la Défense).

\section{Voir infra.}

29. Andrej Andreevič Smirnov (1905-1982), éminent diplomate soviétique, occupa la dignité d'ambassadeur plénipotentiaire extraordinaire. Il collabora dès 1936 à l'appareil central du NKID, ensuite, de 1937 à 1941, à la représentation de l'URSS en Allemagne en tant que conseiller et enfin, comme ambassadeur de l'URSS en Iran de 1941 à 1943. De 1943 à 1949, il fut directeur de la troisième section européenne du NKID (rebaptisé MID en 1946). Il fut en même temps, de 1946 à 1949, adjoint au ministre des Affaires étrangères de 1'URSS, membre du collège de ce même ministère.

30. Décret du SNK de 1’URSS n 1344-402s du 6 octobre 1944.

31. Décret du SNK de l'URSS n 1482-456s du 23 octobre 1944.

32. Le règlement de ce service et le personnel du secrétariat correspondant, y compris les représentants du fondé de pouvoir à l'étranger, furent entérinés par le décret du SNK de l'URSS $\mathrm{n}^{\circ} 1482-456 \mathrm{~s}$ du 23 octobre 1944.

33. GARF, 9526/1/1/21-27. 
SVAG ». Le 25 juin il fut réorganisé en « Secrétariat au rapatriement des citoyens soviétiques et étrangers », et le 7 avril 1947, en «Bureau de rapatriement des citoyens soviétiques et étrangers et de recherche des citoyens des Nations Unies ». Ce bureau fut à son tour rebaptisé le 1 1er juillet 1948 « Bureau de rapatriement et de recherche des citoyens des Nations Unies ». Il fut dirigé pendant tout ce temps par le major général Jurkin ${ }^{34}$. Celui-ci eut pour adjoint dans un premier temps le colonel A. S. Evseev qui dirigeait en même temps le Bureau de rapatriement et de recherche des citoyens de la section soviétique du Conseil de contrôle. Plus tard, c'est le colonel Pisarenko qui fut nommé adjoint. Le colonel M. I. Veršinin fut directeur des opérations jusqu'à ce qu'il remplace le général Davydov dans ses fonctions de représentant plénipotentiaire de la SVAG chargé du rapatriement dans les zones française et américaine en avril 194735 (en 1951 il dirigea la mission soviétique de rapatriement dans la zone française ${ }^{36}$ ).

Dans les administrations militaires américaine et anglaise, les Bureaux des prisonniers de guerre et des personnes déplacées étaient dirigés par Michaelson (auprès du secrétaire Messek) et Kentchington ${ }^{37}$.

\section{Le rapatriement depuis la France}

Dès septembre 1944, une grande quantité de citoyens soviétiques fut rassemblée dans un camp américain près de Verdun. En outre, à la fin de cette même année les Belges et les Britanniques remirent aux Français plusieurs milliers de personnes déplacées. Avant la capitulation il y avait sur le territoire français près de 15000 prisonniers de guerre soviétiques portant l'uniforme de la Wehrmacht ${ }^{38}$.

La France est l'un des rares pays où le rapatriement débuta dès 1944. La mission soviétique de rapatriement y fut ouverte le 10 novembre 1944 et compta une cinquantaine de collaborateurs ${ }^{39}$. Le fait qu'on mit à sa tête le major général Vasilij Mihajlovič Dragun, agent de renseignements de carrière et aide de F. Golikov, montre l'importance qu'on lui attribuait. Dragun avait été nommé aide de Golikov

34. SBZ-Handbuch..., op. cit., p. 51. Le bureau fut liquidé par le décret du Conseil des ministres du 5 novembre 1949 .

35. Communication de Dratvin à Kœnig du 3 avril 1947. Cf. Ministère des Affaires étrangères, Colmar (cité infra MAE/Colmar), Archive de l'occupation française en Allemagne et Autriche, Colmar, 106, Ad IV BC. Veršinin était en même temps représentant de l'URSS au Directorat des prisonniers de guerre et des personnes déplacées du Conseil de contrôle.

36. GARF, $7317 / 20 / 1 / 3,5$.

37. GARF, 7317/20/3/105-109.

38. Cf. le rapport du 19 septembre 1944 de A. Sinjak et M. Mašinin, collaborateurs à la représentation commerciale soviétique à Londres, sur leur mission dans la France libérée (V. N. Zemskov, « Repatrijacija sovetskih graždan i ih dal'nejšaja sud'ba, 1944-1956 » (Le rapatriement des citoyens soviétiques et leur devenir, 1944-1956), Sociologičeskie issledovanija, 5 , 1995, p. 4 (avec une référence à GARF 9526/2/16/12-13).

39. Le quartier général de la mission se trouvait à Paris sur l'avenue Bugeaud et, à partir de 1946, au 4, rue du Général-Appert. 
le 4 octobre, et le 18, il s'envolait déjà par avion spécial pour Paris, passant par Bakou, Bender-Shah, Malte et Marseille. Il était accompagné du major général A. I. Vihorev, des lieutenants-colonels F. I. Mel'nikov et Peregudov et du major P. M. Anan'ev qui prirent la direction des missions soviétiques de rapatriement en Suisse, Belgique, Hollande et dans le nord de la France ${ }^{40}$.

V. M. Dragun était essentiellement le résident et le coordinateur des missions soviétiques de rapatriement en Europe de l'Ouest. Il était pour ainsi dire responsable de tout le «théâtre d'Europe occidentale ». Né en 1898, il rejoignit l'Armée rouge dès 1917 et fit partie des services de renseignements militaires de 1937 à 1947. Pendant la guerre, il dirigea le Bureau exécutif de la direction principale de renseignements de l'Armée rouge et fut l'adjoint du directeur des missions militaires de l'URSS en Grande-Bretagne (1942-1943), en Italie et en France (19441945). Il fut le représentant du Secrétariat au rapatriement en France (ce qui équivaut en fait à toute l'Europe) à partir du printemps de 1945. On lui confia naturellement les missions les plus délicates. Ce fut à lui notamment que Molotov accorda les pleins pouvoirs pour représenter l'URSS aux négociations secrètes qui eurent lieu entre les Américains et le général SS K. Wolf sur la capitulation des forces allemandes en Italie du nord ${ }^{41}$.

Le cumul des activités sur le plan européen et français allait présenter des difficultés : en mars 1945 Dragun fut remplacé par le lieutenant-colonel Berezin à la direction de la mission française de rapatriement. Celui-ci occupa ce poste environ deux ans ${ }^{42}$. Pendant tout ce temps (jusqu'au début de 1947), le groupe situé en France s'occupait de fait du rapatriement depuis les territoires belge, hollandais, luxembourgeois et suisse, et même depuis certaines régions occidentales de l'Autriche (durant l'été et peut-être l'automne 1945) ${ }^{43}$. L'Afrique du Nord se trouvant naturellement sous la «tutelle » de la mission française, il y avait au Maroc un groupe composé du capitaine $\ddot{Y}_{a h o v}$ et du lieutenant-chef $\ddot{Y}_{a b a n o v}{ }^{44}$. De mai à décembre 1947, c'est le colonel N. A. Filatov qui dirigea la mission. Après l'incident de Beauregard, les activités de celle-ci furent interrompues jusqu'en janvier 1951, date à laquelle le lieutenant-colonel Kločkov ${ }^{45}$ en prit la direction.

40. Cf. l'album rassemblé par Mel'nikov sur le rapatriement des citoyens soviétiques depuis la zone du $21^{\mathrm{e}}$ corps d'armée britannique qui avait été déployé au nord-ouest de l'Allemagne, au Danemark et en Belgique. Cf. CMVS (Central'nyj Muzej Vooružennyh Sil Rossijskoj Federacii - Musée central des forces armées de la Fédération de Russie), 3/53490.

41. Cf. la lettre de Molotov à l'ambassadeur des USA en URSS, A. Harriman, du 12 mars 1945 (SSSR i germanskij vopros, 1941-1949: Dokumenty iz Arhiva vnešnej politiki Rossijskoj Federacii (L'URSS et la question allemande, 1941-1949: Documents extraits des Archives de la politique étrangère de la Fédération de Russie), 2 vols, $1: 22$ ijunja 1941-8 maja 1945 (22 juin 1941-8 mai 1945), ed. par G. P. Kynin et J. Laufer, Moscou, 1996, pp. 616-617, avec référence à AVP RF, 06/7/14/137/10).

42. Nous disposons de données indiquant que la mission fut dirigée par le colonel Kolesov en mars 1946 (cf. Archives diplomatiques, Ministère des Affaires étrangères, France (cité infra AD-MAE), Z-Europe 1944-1960, vol. URSS, dossier n 4, p. 38).

43. GARF, 9526/1/7/115.

44. Cf. AD-MAE, Z-Europe, 1944-1960, vol. URSS, dossier n 4, pp. 32-35.

45. Cf. P. M. Poljan, op. cit., p. 386. 
Le réseau français comptait près de 130 lieux de rassemblement et camps de citoyens soviétiques, c'était le plus imposant d'Europe ${ }^{46}$. Selon les données françaises, il y transita près de 110000 personnes $^{47}$ et selon les chiffres soviétiques,

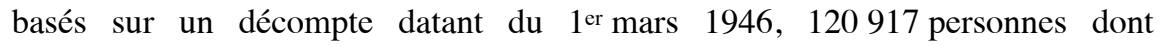
85409 prisonniers de guerre et 35508 civils (comprenant 17463 hommes, 15984 femmes et 2061 enfants) ${ }^{48}$. Le 3 décembre 1946, le nombre de personnes prétendant à la citoyenneté soviétique ayant fortement diminué, la France annonça la fin du rapatriement depuis son territoire ; en fait, celui-ci se prolongea jusqu'en mai 1947, affectant en tout 10481 personnes.

Cependant, le camp soviétique le plus important de France, Beauregard, situé dans la banlieue parisienne entre Versailles et la Celle-Saint-Cloud, poursuivait ses activités, jouissant du bénéfice de l'exterritorialité. Son administration et son système de protection restèrent soviétiques même après 1944. Les officiers du NKVD y faisaient la loi sans aucun contrôle, n'hésitant pas à avoir occasionnellement recours à des attentats ou des enlèvements, puisque le camp disposait même d'un aérodrome. Le 14 novembre 1947 eut lieu un contrôle surprise de ce camp, qui revêtit plutôt la forme d'un raid, à grand renfort de policiers (2 000), de CRS (150) et même de tanks (au nombre de quatre) ${ }^{49}$. Cette opération - déclenchée par les déclarations, le 9 octobre, de Dmitri Spetchinsky, citoyen français émigré de Russie en France en 1922 qui protestait contre l'intention de son épouse, Sofija Subbotina $^{50}$, de se faire rapatrier avec leurs trois enfants âgés de sept à dix ans - eut un résultat positif : on retrouva femme et enfants dans le camp où ils n'avaient même pas été enregistrés. On y découvrit en outre une cache d'armes prises à l'ennemi.

Après cette opération, le groupe du colonel Filatov fut rappelé en URSS sur l'insistance du gouvernement français, et vingt-quatre activistes prosoviétiques ou membres de l' «Union des citoyens soviétiques » furent immédiatement envoyés en Allemagne orientale ${ }^{51}$. Le camp fut mis sous le contrôle des autorités françaises

46. Voir la liste en annexe, pp. 188-189.

47. Cf. G. Coudry, op. cit.; voir également le compte rendu de cet ouvrage de N. Ross dans Russkaja mysl', 4170, 17-23 avril 1997,p. 16.

48. P. M. Poljan, op. cit. p. 276.

49. Les informations surcet incident sont principalement tirées des journaux Le Mondeet l'Humanité.

50. S. Subbotina était la fille de l'amiral Roždestvenskij. Son divorce devait avoir lieu le 21 novembre 1947 à Nice.

51. Dans ce groupe se trouvaient I. A. Krivošein, A. P. Pokotilov, A. A. Ugrimov, N. S. Kačva, etc. Arrêtés le 25 novembre 1947, ils rejoignirent le camp soviétique de Brandenbourg à travers les zones d'occupation occidentale et furent dirigés sur l'URSS en février 1948. Ils furent rejoints par des membres de leurs familles qui quittèrent Marseille sur le «Rossija » à la fin d'avril 1948 (cf. Nina Krivošeina, Četyre treti našej žizni (Quatre tiers de notre vie), Paris, 1984, pp. 164-171). Il semble que l'on ait arrêté Sorokin, collaborateur de Filatov et secrétaire de l'ambassadeur soviétique, à Marseille à la fin de novembre 1947. Les 8 et 9 décembre 1947, Molotov expédia plusieurs notes à Bideau (information communiquée par le docteur Gersdorf). L'URSS répliqua en chassant du pays le colonel Marquet, directeur de la mission française de rapatriement, ainsi que son secrétaire, Dumas, bien que Marquet eût exprimé publiquement sa désapprobation de la politique de son pays en ce qui concerne le rapatriement. Avant leur départ, il rendit avec Dumas une visite d'adieux de dix minutes au lieutenant général Golubev (GARF, 9526/4/36/140). 
à partir du $1^{\text {er }}$ décembre. Le 17 novembre, l'ambassadeur de l'URSS en France, Bogomolov, et à sa suite le ministère des Affaires étrangères de l'URSS protestèrent par voie de notes, et le 18 novembre par une déclaration qui qualifiait l'opération policière de Beauregard de provocation politique et en jugeait les méthodes dignes de la Gestapo. Même le Parti communiste français s'en mêla. Au cours de la première quinzaine de décembre, le lieutenant-colonel Marquet, directeur de la mission française de rapatriement, fut expulsé de Moscou.

Après le rappel de la mission soviétique, les candidats au rapatriement accomplirent les formalités nécessaires auprès du consulat soviétique ou auprès des missions de rapatriement en Allemagne, tout d'abord dans la zone américaine, ensuite dans la zone française. Le camp de Beauregard continua à fonctionner jusqu'en 1951 sous contrôle français. Mais les résultats du rapatriement dans la période 1948-1951 furent plutôt ternes - en tout 218 personnes (sans compter les anciens émigrés russes qui retournèrent en URSS en 1946 et 194752).

Rappelons ici que les officiers soviétiques se heurtèrent, tant en France et en Allemagne que dans d'autres pays, à un fort sentiment d'hostilité envers le rapatriement et, naturellement, aux moyens mis en œuvre pour l'éviter. L'un de ces moyens, typiquement français, était l'enrôlement dans la Légion étrangère, qui, bien que basée en France, avait des centres de recrutement dans d'autres pays comme par exemple l'Allemagne (à Stuttgart) ou l'Autriche (à Innsbruck) ${ }^{53}$. Ajoutons que les citoyens soviétiques, ne pouvant pas officiellement être admis dans la Légion, devaient dissimuler leur nationalité pour pouvoir s'enrôler ${ }^{54}$. Le moyen le plus efficace en France était la protection par les parents s'il y en avait, ou par des organisations non gouvernementales qui s'efforçaient de venir en aide aux personnes se trouvant dans une position trop désespérée ${ }^{55}$. Il arrivait fréquemment que les émigrés de la première vague aident les nouveaux venus en écrivant des lettres de recommandation, en organisant des mariages blancs ou en trouvant d'autres moyens de favoriser la naturalisation de leurs compatriotes. Il existait notamment des organisations actives tels le Bureau central d'aide aux Russes, le Comité d'entraide des Jésuites, le Comité d'émigration Maklakov, l'Amicale ukrainienne de France, etc. ${ }^{56}$.

52. L'ukase du présidium du Soviet suprême du 14 juin 1946 amnistie les émigrés russes blancs pour leur participation à la contre-révolution et propose la citoyenneté soviétique à ceux qui la désirent (selon certaines données, pas moins de 10000 personnes répondirent à l'ukase dans la seule France (Nikita Krivošein, « Moj otec byl 'vol'nym kamenščikom' » (Mon père était « franc-maçon »), Russkaja mysl', 4184, 24-30 juillet 1997,p. 17); cf. aussi les décrets du Conseil des ministres de l'URSS n 1919-809s du 29 août 1946 et n 2747-866s du 2 août 1947. Dans ces décrets il est question d'un contingent de 2000 personnes. En fait, selon l'état du 1 $1^{\text {er }}$ juillet 1947, il partit de France 2827 émigrés ukrainiens et biélorusses, et 267 russes (AVP RF, 082/34/147/14/136).

53. Cf. par exemple K. Eisterer, art. cit.,pp.125-141.

54. L'engagement était d'un minimum de cinq ans et, en plus d'une rémunération, l'on pouvait compter sur une indemnisation de déplacement (AVP RF, 082/35/161/19/100-102).

55. GARF, 9526/1/7/53.

56. GARF, 9526/1/1/170-177, où l'on décrit aussi comment les officiers de la mission russe de rapatriement confisquaient tout bonnement tout document attestant de la citoyenneté française. 


\section{Le rapatriement depuis la zone française d'occupation en Allemagne}

On pourrait penser que, liés comme ils l'étaient à l'URSS par la Conférence de Yalta, l'Angleterre, les États-Unis et la France auraient mené dans les zones qui dépendaient d'eux une politique unique de rapatriement, similaire à celle qu'ils menaient sur leur territoire. Il n'en était rien. En dépit de la similitude dans les directives de départ sur le rapatriement, la politique des administrations des zones occidentales d'occupation se faisait en fait de manière individuelle. De plus, les tâches qui incombaient à ces administrations étaient de proportions variables : si les Britanniques et les Américains, en règle générale, se disputaient la suprématie, les Français, eux, devaient assumer le plus gros des $5 \%$ du flux commun des personnes en voie de rapatriement.

Si l'on compare les Américains et les Britanniques, par exemple, les premiers étaient nettement plus libéraux politiquement et plus souples en pratique. Par exemple, des milliers de personnes désirant décliner l'honneur d'être rapatriées de force se précipitèrent dans les zones américaines (tant en Allemagne qu'en Autriche) comme vers des havres de salut et dans de nombreux cas y trouvèrent effectivement un abri. Selon W. Jacobmeyer, « dans la zone américaine, la prévention contre le rapatriement forcé était plus forte et effective que dans la zone britannique ${ }^{57}$.

Ceci se reflétait dans les hommes et leur conduite. Selon le témoignage de N. Karpov, l'auteur de la nouvelle «Malen'kij ostarbajter» (Le petit STO) qui vécut quelques temps «chez» les Américains et les Britanniques, ces derniers étaient extrêmement corrects mais très froids. « Ils vous regardent sans vous voir, ils vous méprisent. Les Américains, par contre, avaient une attitude entièrement différente: ils s'adressaient à vous avec bienveillance, toujours d'égal à égal, toujours prêts à échanger quelques mots; et on mangeait bien mieux chez eux $»^{58}$.

La France se tenait un peu à part, comme d'ordinaire, mais sa position, rappelons-le, était assez ambiguë étant donné la question des prisonniers originaires d'Alsace-Lorraine. De plus, des communistes participaient au gouvernement mis en place entre la fin de la guerre et 1947. Quoi qu'il en soit, les affirmations de W. Jacobmeyer et de N. Tolstoj selon lesquelles la France, ayant conclu un accord avec l'URSS le 26 juin 1945, observait une position prosoviétique et livrait même des Polonais et des Baltes qui se trouvaient sur les territoires placés sous son contrôle, nécessitent un examen critique ${ }^{59}$. Elles ont été remises en question par

57. W. Jacobmeyer, « Vom Zwangsarbeiter zum heimatlosen Ausländer. Die Displaced Persons in Deutschland. 1941-1945 », Kritische Studien zur Geschichtswissenschaft, 65, 1985, p. 134. Ce qui ne les empêcha pas par ailleurs d'interrompre le ravitaillement des personnes déplacées russes vivant à l'intérieur des camps de citoyens soviétiques dès le 1 er décembre 1945.

58. Communication orale, 17 juillet 1995.

59. Selon Jacobmeyer, il existe un cas de rapatriement en Pologne en octobre 1945 d'un convoi de Volksdeutsche qui provoqua une protestation officielle de la part de l'UNRRA (W. Jacobmeyer, art. cit., pp. 142-143). En 1950 il y eut même un débat dans la presse sur d'éventuelles exécutions de personnes déplacées qui auraient eu lieu pendant l'été de 1945 dans un sous-sol de la mission soviétique à Stuttgart-Zuffenhausen (qui se trouvait à l'époque en zone française). 
K. Eisterer - du moins en ce qui concerne la zone française en Autriche. Nous avons quant à nous pris connaissance d'un document qui soutient les accusations de Jacobmeyer et Tolstoj. Le 21 août 1945 le lieutenant-colonel Pelik communiquait à D. A. Filin, directeur du Bureau des prisonniers de guerre et des personnes déplacées de la partie soviétique de la Commission alliée en Autriche :

« La situation est plus satisfaisante dans la zone française d'occupation. Il y a un camp de citoyens soviétiques à Landeck (à $72 \mathrm{~km}$ à l'ouest d'Innsbruck); 180 personnes ont quitté le camp et 300 autres attendent d'être prochainement dirigées sur l'URSS. Le commandement français se conduit de façon loyale envers nos représentants, les aide en informant et en regroupant les citoyens soviétiques et participe de bon gré à la détention et à la livraison des éléments antisoviétiques.

Dans la zone française, on peut porter à 5000 le nombre de personnes qui ne retournent pas en URSS - Lettons, Lituaniens, Estoniens. Ils s'abritent tous dans les camps de l'UNRRA [United Nations Relief and Rehabilitation Administration] dont les représentants ont déclaré que ces personnes ne sont pas soumises au rapatriement soi-disant parce que la citoyenneté soviétique se définit pour ces nationalités par les frontières de 1938. Le capitaine Kulikov retient prisonniers des activistes antisoviétiques (Kričevskij, etc.) avec l'aide de la police française. De plus, se cachent ici des antisoviétiques tels le général Polozov, le colonel Poltavec-Sotranica, le chef de l'armée cosaque du Terek, le colonel Kulakov (tous des Russes blancs). Selon le capitaine Kulikov, les autorités françaises ont consenti à nous les livrer. »60

L'attachement de la France aux principes de Yalta correspond avec la présence au pouvoir du premier cabinet de de Gaulle (c'est-à-dire jusqu'au début de 1946). Mais plus tard, lorsque l'URSS reprocha inlassablement à tous les Alliés de ne pas respecter les accords de Yalta, elle ne manifesta aucune reconnaissance à la France $^{61}$. Le rapport qui suit est typique :

«Les autorités locales françaises d'occupation qui gèrent le rapatriement font semblant de prendre toutes les mesures et de tout faire pour en accélérer le processus, mais en réalité leur activité contredit l'accord du ministère soviétique des Affaires étrangères du 28 avril 1948 et ralentit le rapatriement des citoyens soviétiques déplacés. ${ }^{62}$

Cf. par exemple O.L., «Ešče odna Katyn'. Sovetskaja repatriacionnaja missija podozrevaetsja v massovom rasstrele Di-Pi » (Un autre Katyn. La mission de rapatriement russe est soupçonnée d'exécution collective de personnes déplacées), Russkaja mysl', 23 juin 1950.

60. GARF, 9526/2/56/46-47.

61. Voir par exemple GARF 9526/1/1/164, 170-178 et passim.

62. Rapport du colonel Gorelov sur les activités dans la zone française d'occupation en juillet et août 1948 (AVP RF, 082/35/161/16/3). 
De plus, selon certains témoignages, les officiers français faisaient souvent preuve de plus de libéralisme et d'humanité que les Américains eux-mêmes lorsqu'ils devaient prendre des décisions concrètes sur les personnes ${ }^{63}$.

Selon les données françaises, en novembre 1945, 101459 citoyens soviétiques avaient été rapatriés depuis la zone d'occupation en Allemagne, et sur les 77261 personnes déplacées qui y restaient à cette époque, il y avait 2450 citoyens soviétiques à proprement parler (venaient s'ajouter des Ukrainiens soviétiques (313), des Estoniens (828), des Lettons (1 334), des Lituaniens (1 594), des Volksdeutsche baltes (1 834); la majorité du groupe était formée de Polonais (59 957)). Vers la fin de 1946 le nombre de citoyens soviétiques rapatriés atteignit 153000 , et pour le reste des années 1940, il augmenta de 314 personnes, ce qui est égal à son augmentation entre 1951 et 1952. Pour le moins, 458 Soviétiques (sans compter 737 Estoniens, 2605 Lettons et 2523 Lituaniens) fuirent le rapatriement et émigrèrent pour la plupart aux États-Unis (155), en France (142), et au Canada (87) ${ }^{64}$.

De plus, ajoutons que jusqu'à la fin de 1948 il n'y avait pas de mission soviétique de rapatriement à proprement parler dans la zone française et que tout le rapatriement passait officiellement par la mission située dans la zone américaine (à Francfort-sur-le-Main), mission dont dépendaient les officiers en activité dans la zone française. Leur quartier général se trouvait au début à Rastadt ${ }^{65}$, près de Baden-Baden, « capitale » de la zone française.

À la fin de février 1946, un groupe d'officiers soviétiques fut accrédité pour travailler dans la zone française : le capitaine Razdobudko à Oberstein (Rhénanie), le capitaine Smirnov à Tübingen, et le sous-lieutenant Konovalov à Rottweil (Wurtemberg) ${ }^{66}$. Le 7 mai 1946, après la fermeture du camp de réfugiés de Stetten, les Soviétiques en voie de rapatriement qui y avaient été placés furent transférés à Immendingen 67 .

Le 8 février 1946, le général Davydov, représentant soviétique principal dans les zones française et américaine, exigea des autorités françaises des listes des citoyens soviétiques qui se trouvaient dans la zone d'occupation française. Le général Kœnig s'apprêtait à établir ces listes lorsque, le 13 février, le général Laffon engagea une correspondance avec lui qui remettait en cause la nécessité d'y

63. Nous ne devons pas oublier que c'est en France que s'est formée la Légion étrangère, qui intégra de nombreuses personnes déplacées. La « chasse » aux légionnaires eut lieu non seulement sur le territoire français mais aussi en Afrique du Nord.

64. Haut Commissariat de la République française en Allemagne. Service des Personnes Déplacées. Sept ans d'activité en faveur des personnes déplacées en zone française d'occupation. 1945-1952, s.1.n.d.

65. Adresse : Frembergenstrasse, 39 (MAE/Colmar, 106, Ad IV Bc).

66. Communication de Laffon à Kœnig du 12 mai 1945 (MAE/Colmar, ADM-105, ABM IV $\mathrm{Ba})$.

67. Immendingen (ne pas confondre avec Emmendingen près de Fribourg!) se trouve à $10 \mathrm{~km}$ de Tütlingen (près de Donaueschingen). Près du lieu de rassemblement soviétique on plaça des centaines de personnes en voie de rapatriement à l'hôtel Falken. Ajoutons que les relations entre les représentants soviétiques et le lieutenant Levi, commandant du camp, étaient bonnes (Cf. la lettre de Laffon à Kœnig du 12 mai 1945 (MAE/Colmar, ADM-105, ABM IV Ba)). 
inclure les personnes ayant vécu sur le territoire polonais d'avant guerre ${ }^{68}$. Plus tard, en juillet, les femmes soviétiques mariées ou fiancées avec des militaires des forces alliées furent aussi exemptées du rapatriement.

Le 12 mars, le lieutenant-colonel Bancirev (adjoint de Davydov dans la zone française) demanda au colonel Poignant, directeur du Bureau français des affaires des personnes déplacées, d'accélérer le processus de rapatriement des 1010 citoyens soviétiques logés chez des particuliers ${ }^{69}$.

Le rapatriement des citoyens soviétiques faillit s'interrompre au tout début de l'automne 1946. Le 4 septembre, le général de Villeneuve, directeur du bureau des missions étrangères sous les ordres de Kœnig, convoqua le major général Davydov à Baden-Baden et lui annonça que si l'activité de la mission française en zone soviétique continuait à être bloquée comme elle l'était depuis un mois, la mission soviétique en zone française subirait le même sort70.

Le 8 octobre 1946 au quartier général de l'UNRRA à Arolsen eut lieu, sur l'initiative de Davydov et sous la présidence du général Greenslade, une réunion sur le rapatriement des citoyens soviétiques avec la participation des dirigeants de l'UNRRA de chaque zone (le général Lenclud pour la zone française). Davydov indiqua que les résolutions $n^{\circ} 71,92$ et 99 prises à ce sujet par l'UNRRA n'étaient pas exécutées; que l'UNRRA soutenait même des criminels de guerre comme l'avait montré l'incident avec Aranasevič et Demidov, deux ouvriers du camp de l'UNRRA à Brombach, anciens membres de l'administration allemande en Biélorussie et maintenant arrêtés. En témoignait aussi la remise par le représentant de l'UNRRA à Biberach d'un certificat d'apatride au général tsariste Lampe. Un accord de principe sur le regroupement par nationalités des personnes déplacées fut conclu ${ }^{71}$.

Deux semaines plus tard (le 22 octobre), eut lieu à Haselach (zone du général Lenclud) une autre réunion à laquelle participèrent, outre Davydov et Bancirev, le major Akopov et le lieutenant Konovalov. À l'ordre du jour il y avait l'accélération du rapatriement pour laquelle Davydov mit en avant toute une série d'exigences, dont la garantie que les officiers soviétiques pourraient pénétrer librement dans les camps $^{72}$ et participer à l'enregistrement des citoyens soviétiques dans la zone française; il demandait aussi la création, en un mois, d'un camp réservé aux citoyens soviétiques et réclamait de l'aide pour faire de la propagande dans les

\section{Ibid.}

69. MAE/Colmar, ADM-105, ABM IV Ba.

70. GARF, 7317/20/64/141-149. L'ordre correspondant fut émis par Kœnig mais, à notre connaissance, ne fut jamais mis à exécution.

71. MAE/Colmar, ADM-105, ABM IV Ba.

72. Conformément à l'ordonnance de Kœnig à laquelle l'UNRRA devait se plier, seuls le major Roche, directeur du Bureau de rapatriement de l'administration française d'occupation et le lieutenant Cerise avaient plein pouvoir en ce qui concerne l'accompagnement des officiers soviétiques dans les camps. Leurs «nombreuses occupations » leur fournirent un bon prétexte pour barrer l'accès aux camps (voir les minutes de la réunion de Haselach du 22 octobre 1946, GARF, 7317/20/64/141-149). 
camps en faveur du rapatriement et interdire toute propagande contraire sous forme orale ou écrite.

Enfin, le 4 juin 1947, comme pour faire écho à la réunion des ministres des Affaires étrangères des pays alliés qui se tint à Moscou le 23 avril 1947, eut lieu une autre réunion avec le côté français. Y prirent part le major Roche, le général Basilov (adjoint de Golikov, venu de Moscou en inspecteur), le général Veršinin (responsable du rapatriement depuis l'Allemagne) et le colonel Gorelov (son représentant dans la zone française ${ }^{73}$ ). Basilov exposa sa vision du problème, qui d'ailleurs ne présentait rien de nouveau si ce n'est quelques références aux décisions prises par Moscou sur l'accélération du rapatriement. Les mesures proposées avaient un aspect de déjà vu - simplification de l'accès aux camps pour les officiers soviétiques, permission de faire de la propagande en faveur du rapatriement et, en même temps, interdiction de la propagande contraire, mise à exécution de la proposition de tri des réfugiés par nationalités. Sur un plan plus concret, Basilov exigea qu'on lui remette des listes complètes des camps de Bad Kreuznach, Würnach et Tübingen, qu'on accélère le convoi en provenance d'Immendingen et que soit accrédité le capitaine Savel'ev à Coblence. Roche, quant à lui, donna à Gorelev une liste de dix-huit rapatriés volontaires et déplora le fait que le côté soviétique ne respectait pas la résolution de l'ONU du 12 février 1946 sur les réfugiés et celle adoptée à la session de l'UNRRA à Genève en présence du général Davydov ${ }^{74}$.

Le 5 juillet 1947, le lieutenant général Dratvin écrivit une lettre au général divisionnaire Noiret, adjoint de Kœnig, dans laquelle il mentionnait la dissolution de l'UNRRA et le transfert des pleins pouvoirs de celle-ci à l'IRO (International Relief Organization), et demandait l'accréditation de dix officiers soviétiques supplémentaires dans la zone française ${ }^{75}$. Et le 12 novembre, il s'adressa directement à Kœnig, lui demandant de sanctionner la visite d'une délégation de 34 travailleurs sociaux pour une durée de deux semaines dans la zone française ${ }^{76}$.

Ainsi, la mission soviétique de rapatriement s'ouvrit-elle dans la zone française en Allemagne entre la fin de 1948 et le début de 1949, un peu plus de trois ans après les missions correspondantes en $《$ Bizonie $\gg 77$ et en Autriche qui avaient été ouvertes entre août et décembre 1945. Ceci était vraisemblablement lié au caractère sérieux des menaces américaines de fermer la mission de Francfort-sur-le-Main qui

73. En mars 1947, Veršinin posa la question de l'accréditaion du lieutenant colonel Gavrilov dans cette fonction. Cf. communication de de Rosen à Kœnig du 12 mars 1947, MAE/Colmar, 106, Ad IV Bc.

74. Cf. compte rendu du 6 juin 1947, MAE/Colmar, ADM-105, ABM IV Ba.

75. MAE/Colmar, ADM-105, ABM IV Ba.

76. Lettre de Dratvin à Kœnig du 12 novembre 1947, MAE/Colmar, 106, Ad IV Bc.

77. Étaient ainsi nommés, même dans les documents officiels, les territoires occupés par les Britanniques et les Américains. Leur consolidation aboutit dans la formation de la Bizonie pendant l'automne 1946. En avril 1948, à la suite de longues hésitations et de négociations peu fructueuses avec la SVAG, la zone française fut rattachée à la Bizonie qui devint la « Trizonie » (curieusement, pendant les premières étapes des négociations entre l'URSS, les USA et l'Angleterre sur l'avenir de l'Allemagne, celle-ci était souvent nommée « Trizonie »). 
avait jusque-là simultanément couvert les zones américaine et française. (À partir de mars 1949, la situation se renversa et la zone américaine (l'anglaise à partir de mars 1950) desservit les lieux de rassemblement de la zone française.)

Le colonel R.S. Pavlin en fut le premier directeur soviétique officiel. En novembre 1949 il fut remplacé par le colonel V. I. Arunov qui avait dirigé la mission de Francfort-sur-le-Main jusqu'à sa fermeture ${ }^{78}$. Le quartier général de la mission ne se trouvait plus à Rastadt mais à Baden-Baden. Il y avait au début 28 camps de regroupement sur le territoire de la zone.

Incidemment, le «volume de travail » à proprement parler dans la zone française était inférieur à celui des zones américaine et britannique. Ainsi, selon les estimations soviétiques, en novembre 1947 il y avait au total dans la zone française 9650 personnes susceptibles d'être rapatriées ${ }^{79}$.

Les lieux de rassemblement fermèrent les uns après les autres et furent d'abord remplacés par des foyers à Fribourg, Ravensbourg et Tübingen qui, avec le temps, subirent le même sort. L'un des derniers - sinon le dernier - de ces lieux se trouvait à Emmendingen. Il fut décidé au printemps ou en été 1952 de le transférer à Baden-Baden mais le tranfert fut différé indéfiniment bien que le camp ne fût plus entretenu (il y restait en tout huit paires de draps) ${ }^{80}$.

Cependant, il passait encore par ce camp un nombre limité de personnes, mais qui était tout de même supérieur à 8 par mois : ainsi, 29 personnes furent-elles rapatriées en mai et juin 1952, dont 6 en provenance de la zone française, 19 de la zone américaine, et 4 de France ${ }^{81}$, et en juillet et en août 1952, 16 personnes, dont 6 en provenance de la zone américaine, 1 de la zone britannique, 1 de la zone française, et 8 de France ${ }^{82}$.

Mais il était clair que le rapatriement était destiné à s'arrêter là, comme le confirma la signature de deux documents à Bonn et Paris les 26 et 27 mai 1952 : un accord militaire entre les gouvernements américain, britannique et français d'un côté et ouest-allemand de l'autre, et un accord sur la «Communauté de défense européenne ».

En juillet 1952, les troupes françaises d'occupation interdirent l'accès à certaines régions du nord de la zone aux officiers soviétiques, et le 24 octobre 1952, Courtois, directeur du Bureau des personnes déplacées, informa Veršinin que le service français aux réfugiés serait fermé à partir du 1er novembre et qu'il devrait dorénavant, en tant que chef de la mission soviétique, contacter les autorités fran-

78. En novembre 1951, c'est le colonel M. I. Veršinin qui en devint le directeur, après avoir été à la tête du bureau exécutif du Bureau du rapatriement de la SVAG.

79. AVP RF, 082/35/160/14/6-8. Les officiers supérieurs français chargés du rapatriement étaient le colonel Chérifi et le major de Rosen.

80. Nous avons glané ces renseignements dans les rapports de nos représentants chargés du rapatriement des citoyens soviétiques depuis les zones d'occupation en Allemagne en mai et juin 1952, compilés par le colonel Kašin, assistant du directeur du bureau exécutif le 18 août 1952 (AVP RF, 082/40/257/25/45-52).

81. Ibid.

82. AVP RF, 082/40/257/25/60-62. 
çaises par l'intermédiaire du docteur Melon, conseiller du haut-commissaire de la République française en Allemagne (ou Klein, son adjoint), l'officier français de liaison à Baden-Baden étant toujours Trouin, l'ancien représentant à Emmendingen. Curieusement, à cette époque, l'emplacement du lieu de rassemblement de Baden-Baden n'avait pas encore été désigné, mais l'on avait promis qu'il le serait pour le 10 novembre $1952^{83}$.

Tout cela aussi fut organisé selon les conditions traditionnelles, surtout en ce qui concerne le retour des Alsaciens et des Lorrains ainsi que des dépouilles des pilotes français de l'escadrille « Normandie ${ }^{84}$. Soulignons cependant que la zone française d'occupation a servi de passage à ceux qui voulaient retourner en URSS plus longtemps que les autres zones ${ }^{85}$.

\section{Le rapatriement depuis la zone française d'occupation en Autriche}

En ce qui concerne les zones occidentales d'occupation en Autriche, il s'y trouvait, selon l'état du 1 ${ }^{\text {er }}$ mars 1946, environ 53000 personnes et, vers le début de 1952, près de 19000 , que l'URSS s'obstinait à considérer comme ses citoyens ${ }^{86}$. Ceci ne signifie pas pour autant que la différence entre ces deux chiffres désigne le nombre de personnes qui avaient voulu se faire rapatrier : en vérité leur nombre ne dépassait même pas les 2500 , tant en Autriche qu'en Allemagne, la «prise » principale ayant eu lieu en 1945, surtout pendant les mois d'été.

Le premier groupe affecté au rapatriement sur le territoire autrichien fut créé en février 1945 au même titre que les six autres groupes du Secrétariat au rapatriement qui devaient travailler de concert avec l'Armée rouge qui avançait. Composé de 11 personnes, il était dirigé par le lieutenant-colonel Fedorov ${ }^{87}$.

L'opération retentissante que fut le rapatriement des cosaques et des Caucasiens à la fin du mois de mai et au début du mois de juin 1945, selon toute vraisemblance, ne pouvait être que l'œuvre des forces du NKVD et du SMER Ÿ (Smert' špionam). Mais s'il y eut une participation quelconque d'officiers affectés au rapatriement à cette opération, cela ne put être que celle des officiers du $3^{e}$ front ukrainien du maréchal Tolbuhin ${ }^{88}$.

83. Renseignements sur la zone française d'occupation en Allemagne, 9 octobre 1952 (AVP RF, 082/40/257/25/127-130).

84. Renseignements sur la zone française d'occupation en Allemagne, 7 août 1952 (AVP RF, 082/40/257/25/60-62).

85. La date officielle de sa fermeture n'est pas connue précisément.

86. Cf. P. M. Poljan, op. cit., p. 270. En outre, près de la moitié des personnes « prises au filet » se trouvait dans la zone américaine.

87. Ibid., p. 384.

88. Ibid., pp. 237-250. 
Le 7 juin 1945 fut créé le Secrétariat au rapatriement auprès du Conseil militaire du groupe central des armées dont le quartier général était à Vienne ${ }^{89}$. Il semblerait que les renseignements sur les 50 lieux de rassemblement ouverts dans les zones occidentales d'occupation en Autriche pour assurer le rapatriement ${ }^{90}$ datent de cette période.

En août 1945, des membres de ce Secrétariat, dirigés par le colonel de la garde Ikonnikov ${ }^{91}$, furent dépêchés à Salzbourg, Klagenfurt et Innsbruck. Il leur incombait, outre le rapatriement depuis l'Autriche, d'assurer le transit par l'Autriche et la Tchécoslovaquie des rapatriés en provenance de la France, de la Suisse, du sud de l'Allemagne et même, en partie, du sud-ouest de la Tchécoslovaquie. Mais, depuis le mois de juillet, il y avait en Autriche des représentants de la mission de rapatriement du major général Dragun basée en France qui faisaient le même travail. L'on put donc observer, cet étélà, une concurrence insolite entre les « Parisiens » et les « Viennois ».

Lorsque le groupe du colonel Ikonnikov arriva à Salzbourg (zone américaine) au milieu du mois d'août, les 15 personnes de la représentation de Dragun étaient accréditées depuis le mois de juillet 1945. Elles avaient à leur tête deux officiers de liaison, anciens prisonniers de guerre, le capitaine Komarov et le lieutenant-chef Ušakov. Selon le rapport du lieutenant-colonel V. P. Pelik, cette représentation était formée de fainéants et d' « anciens prisonniers de guerre échus là par hasard » qui depuis des mois ne songeaient qu'à servir leurs propres intérêts aux frais des Américains. Par contre, Pelik fournit une évaluation plus positive du groupe du major Vartašesov (il se serait agi d'un groupe de 10 personnes en poste au lieu de rassemblement de Salzbourg; Vartašesov était lui-même un ancien prisonnier). D'ailleurs, même le personnel du groupe d'Ikonnikov ne lui donnait pas entière satisfaction ${ }^{92}$. Le rapport montre aussi clairement qu'il y avait à Salzbourg un autre groupe qui s'occupait clandestinement du rapatriement, le « groupe de Korolev », qui refusait de se soumettre à Ikonnikov et qui « se distinguait par sa brutalité et son manque d'expérience »93.

89. Comme à Berlin, son personnel comprenait 79 personnes. Le directeur en était le major général Fomin, et c'est le lieutenant-colonel Moškov qui en dirigeait le bureau des opérations (GARF, 9526/2/56/95-97) ; cf. également K. Eisterer, art. cit., pp. 130-134.

90. Plus tard, en guise d'autocritique, il sera mentionné dans le rapport de 1952 sur l'activité globale du Secrétariat au rapatriement entre 1946 et 1952 que, lors du retrait convenu des troupes soviétiques au-delà de la ligne de démarcation autrichienne dans la région de Linz, il restait plusieurs milliers de citoyens soviétiques sur le territoire passé aux Américains (GARF, 9526/1/7/157).

91. Ses adjoints étaient, semble-t-il, le colonel Piterskij et le lieutenant-colonel Pelik (GARF, 9526/2/56/95-97).

92. Cf. notamment le rapport du lieutenant-colonnel Pelik sur l'activité du groupe d'Ikonnikov à Salzbourg du 13 au 20 août 1945 qu'il présenta le 21 août 1945 au colonel Filin, directeur du Bureau des prisonniers de guerre et des personnes déplacées (GARF, 9526/2/56/41-46). Le rapport fait mention de la réception glaciale de la part des autorités américaines (de la part notamment du fondé de pouvoir américain, le lieutenant-colonel Stevens).

93. Cf. par exemple ce qui suit : « Le major Lyskov (du groupe de Korolev) choisit une femme du groupe de Komarov (c'était la compagne de l'un des employés de ce dernier), l'envoya en mission dans le camp de Parsch où elle fut immédiatement démasquée car elle y était déjà connue depuis longtemps. Il se répandit immédiatement parmi les personnes ne voulant pas du rapatriement la rumeur qu'un groupe de Tchekistes était arrivé à Salzbourg et s'était installé dans le lieu de rassemblement soviétique. Dans les quelques jours qui suivirent cette nouvelle, le flux vers ce lieu s'interrompit jusqu'à ce que nous trouvions de nouveaux quartiers » (GARF, 9526/2/56/45). 
La zone française elle-même connaissait une situation analogue : «Paris » était représenté par le groupe du capitaine Kulikov94 (remplacé ensuite par le capitaine Baranov) et «Vienne » par le groupe de Pelik ${ }^{95}$. Une solution s'imposait : réduire le personnel et mettre fin aux activités parallèles des divers groupes en soumettant ceux-ci à une direction unique ${ }^{96}$.

Les différentes missions soviétiques de rapatriement des zones occidentales de l'Autriche s'ouvrirent à la fin de 1945 (la première, un groupe de cinq personnes, dans la zone britannique le 15 novembre) et plus tard dans les zones française ${ }^{97}$ (un groupe de dix personnes le 1er décembre) et américaine (dix personnes le 17 décembre). Le quartier général de la mission de la zone britannique était installé à Bruck, à l'hôtel Bauer, et les groupes opérationnels étaient basés à Bruck, Graz et Klagenfurt.

Leur activité souffrait toujours autant d'un manque de coordination et les directeurs se succédaient avec une rapidité étonnante. On compte cinq représentants pour les zones britannique et américaine et sept pour la zone française, lieutenantscolonels ou colonels: B. A. Piterskij, R. F. Rogozinskij, M. P. Minin, F. M. Galika, V V. Mitrofanov, I. H. Venec et V. A. Osokin ${ }^{98}$.

Dans une note adressée au ministère des Affaires étrangères le 13 mai 1947 et dans un communiqué diffusé le 27 mai, l'ambassade d'URSS en France fit état d'obstacles rencontrés dans la zone française d'occupation en Autriche en ce qui concerne le rapatriement de 10000 à 12000 Soviétiques dont 7000 Arméniens qui s'y trouvaient, notamment dans les camps de Kufstein et Landeck. Le 4 juin, le ministère répondit qu'il y avait en fait 4947 Ukrainiens d'origine polonaise, 1244 ressortissants des pays baltes et 296 réfugiés en provenance d'autres régions de l'URSS actuelle.

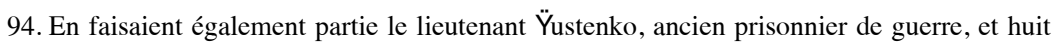
membres du personnel de service (GARF, 9526/2/56/44-46).

95. K. Eisterer, art. cit., p. 132.

96. Des éléments d'activité parallèle et de concurrence pouvaient également s'observer dans la zone britannique, où, après la livraison de cosaques en mai et juin on ne vit plus aucun officier soviétique chargé du rapatriement. Et le major Turasov, qui avait été envoyé dans cette zone le 17 août par Ikonnikov, fut contraint de la quitter dès le 19 car la durée de son visa ne lui permettait pas de séjourner en Carinthie et en Styrie, zones occupées par les Britanniques, où d'ailleurs il put, ainsi que ses collaborateurs le major Lyskov et le sous-lieutenant Berezin, se faire une idée de l'ampleur de la tâche à accomplir (entre10 000 et 12000 personnes). Il faut croire que ce contingent avait été compris dans les comptes du lieutenant-colonel Martynov (membre, apparemment, du groupe de Dragun), qui avait même, une semaine auparavant, le 13 août, conclu un accord avec les Britanniques à Leoben sur la livraison de 9000 à 10000 citoyens soviétiques au poste intermédiaire de Neunkirchen. Cf. la communication de Filin à Želtov du 14 septembre 1945 (GARF, 9526/2/56/70). Cet « accord » souleva la colère d'Ikonnikov qui n'en avait pas été informé. Cf. la communication d'Ikonnikov à Fomin du 26 août 1945 (GARF, 9526/2/56/79).

97. L'adresse de la représentation était : Innsbruck, Herrengasse, 1. Les Français pensaient que le rapatriement s'était conclu en septembre 1945 avec la livraison des derniers citoyens soviétiques du camp de Landeck (cf. K. Eisterer, art. cit., pp.130-141).

98. Pour plus de détails, cf. P. M. Poljan, op. cit., p. 384. 
Par rapport aux missions analogues situées dans les zones américaine et britannique, la mission soviétique de rapatriement d'Innsbruck, dirigée par le colonel Rogozinskij, jouissait dans l'ensemble d'une position plutôt privilégiée : les officiers soviétiques avaient non seulement accès à leurs concitoyens, mais aussi aux ressortissants d'autres nationalités et pouvaient informer les réfugiés de leurs visites sur les ondes et par voie de presse. Ils participaient même librement aux travaux des commissions mixtes chargées de déterminer la nationalité des personnes déplacées. Le chiffre global de citoyens soviétiques rapatriés depuis le 10 juillet 1945 s'élevait à 7504 personnes dont 6300 venant du Vorarlberg et 1204 du Tyrol.

La fermeture des missions soviétiques de rapatriement en Autriche est celle qui dura le plus longtemps : la première à fermer fut celle de la zone américaine en juin 1951. Son activité n'avait d'ailleurs pas été continue, puisqu'elle avait par trois fois au moins officiellement interrompu ses activités ${ }^{99}$. La mission de la zone britannique partit en août 1951 sur les instances des autorités anglaises et non de son plein gré. C'est la mission de la zone française qui fut la dernière à fermer, en mai 1952.

C'est depuis les zones françaises d'occupation en Allemagne et en Autriche que le rapatriement officiel dura le plus longtemps. Ainsi, selon les données du ministère de l'Intérieur autrichien pour le 1er septembre 1951, il s'y trouvait 953 personnes déplacées soviétiques dont 288 Ukrainiens, 165 Russes, 163 Lettons, 141 Lituaniens, 92 Estoniens et 104 représentants d'autres nationalités. Dans ce groupe, les officiers soviétiques connaissaient 330 personnes par leur nom. Sur ce nombre, 140 se trouvaient dans des camps et 190 étaient logées à l'extérieur. Ils eurent des entretiens avec 230 personnes dont 55 étaient favorables au rapatriement, 133 hostiles, et 42 « vivaient avec des étrangers dont elles avaient des enfants » (en d'autres termes, elles étaient favorables au rapatriement mais ne voulaient pas partir) ${ }^{100}$.

Dans la mesure où, selon les Soviétiques, les Alliés positionnés en Autriche cherchaient par tous les moyens à retarder ou à faire échouer toute solution du problème des réfugiés, l'URSS proposa en avril 1948 de transférer toutes les opérations relatives au rapatriement directement au gouvernement autrichien. Cette proposition fut rejetée le 23 avril 1948 par le comité exécutif de la Commission alliée ${ }^{101}$.

Malgré le nombre restreint de départs de réfugiés depuis les zones françaises d'occupation pendant la dernière étape du rapatriement (1946-1952), c'est tout de même dans ces zones, contrairement aux zones américaines et britanniques, que le nombre de rapatriés était supérieur à celui des personnes déclarées hostiles au rapa-

99. Entre 1946 et 1951 , la mission fut quatre fois admise sur le territoire de la zone, du 5 janvier au 2 avril 1946, du 14 janvier au 15 mai 1947, du 7 février (?) au 7 mars 1948 et du mois de mai 1948 au 9 juin 1951 (avec accréditation du personnel de la mission tous les 45 jours); chaque fois la mission se retirait brutalement (GARF, 9526/1/7/1920).

100. AVP RF, 082/40/257/26/4-5.

101. AVP RF, 082/35/161/17/54-57. 
triement. Selon d'autres indices, l'efficacité du rapatriement depuis les zones françaises avait été assez grande. Pendant la dernière étape du rapatriement, alors que les missions soviétiques fermaient les unes après les autres dans les zones américaine puis britannique en Allemagne (et même sur le territoire français), l'importance de la zone française en Allemagne en tant que « principale porte de sortie de l'Europe » s'accrut brutalement. On peut faire le même constat, quoiqu'à un degré moindre, pour la zone française en Autriche.

(Traduit du russe par Christine Colpart)

Rossijskaja akademija nauk

Institut geografii

Staromonetnyj per. 29

$109017 \mathrm{Moscou}$

e-mail :polian@aha.ru 


\section{Liste des camps de rapatriement soviétiques en France}

\begin{tabular}{|c|c|c|c|c|c|}
\hline 1 & L'Ardoise & Gard & 31 & Theix & Morbihan \\
\hline 2 & Banassan & Gard & 32 & Auch & Gers \\
\hline 3 & Mende & Lozère & 33 & Bergerac & Dordogne \\
\hline 4 & $\begin{array}{l}\text { Corbie } \\
\text { Fouilloy }\end{array}$ & Somme & 34 & $\begin{array}{l}\text { Angoulême/Le } \\
\text { Basseau-Narsac }\end{array}$ & Charente \\
\hline 5 & Amiens & Somme & 35 & Isle-sur-Tarn & Tarn \\
\hline 6 & Châteauneuf & Cher & 36 & Labruguière & Tarn \\
\hline 7 & Versailles & Seine-et-Oise & 37 & Saint-Pons & Ardèche \\
\hline 8 & $\begin{array}{l}\text { (Beauregard) } \\
\text { Vendôme }\end{array}$ & Loir-et-Cher & 38 & Caylus & $\begin{array}{l}\text { Tarn-et- } \\
\text { Garonne }\end{array}$ \\
\hline 9 & Bourbonne & Haute-Marne & 39 & Couiza & Aude \\
\hline & (-les-Bains) & & 40 & Lille & Nord \\
\hline 10 & Provins & Seine-et-Marne & 41 & Montbazon & Indre-et-Loire \\
\hline 11 & Meaux & Seine-et-Marne & 42 & Courpierre & Puy-de-Dôme \\
\hline 12 & Paris (Reuilly) & & 43 & Toulouse & Haute-Garonne \\
\hline 13 & Bourg-Lastic & Puy-de-Dôme & 44 & Albi/hôpital & Tarn \\
\hline 14 & Nantiat & Haute-Vienne & 45 & Cherbourg & Manche \\
\hline 15 & La Courtine & Creuse & 46 & Epernon & Seine-et-Oise \\
\hline 16 & $\begin{array}{l}\text { Saint-Amand- } \\
\text { Montrond }\end{array}$ & Cher & 47 & Paris/hôpital & \\
\hline 17 & Fort de Chapoly & Rhône & 48 & Villevaudé & Seine-et-Marne \\
\hline & (Lyon) & NIIOII) & 49 & Lyon & Rhône \\
\hline 18 & Saint-Etienne & Loire & 50 & Reims & Marne \\
\hline 19 & Valence & Drôme & 51 & Saint-Thégonnec & Finistère \\
\hline 20 & Grenoble & Isère & 52 & Commercy & Meuse \\
\hline 21 & Le-Puy & Haute-Loire & 53 & Marseille $\left(7^{\mathrm{e}}\right)$ & Bouches-du- \\
\hline 22 & Marseille & $\begin{array}{l}\text { Bouches-du- } \\
\text { Rhône }\end{array}$ & 54 & Vadenay & Marne \\
\hline 23 & Lannemezan & Hautes- & 55 & Rennes & Ille-et-Vilaine \\
\hline & & Pyrénées & 56 & Le Touquet & Pas-de-Calais \\
\hline 24 & Castres & Tarn & 57 & Besançon & Doubs \\
\hline 25 & Albi & Tarn & 58 & Pontoise & Seine-et-Oise \\
\hline 26 & La Planète & Hautes- & 59 & Châteaudun & Eure-et-Loire \\
\hline & & Pyrénées & 60 & Tours & Indre-et-Loire \\
\hline 27 & Eaunes & Haute-Garonne & 61 & Metz & Moselle \\
\hline 28 & Saint-Junien & Haute-Vienne & 62 & Brive(-la- & Corrèze \\
\hline ת & Nîmes & Gard & & Gaillarde) & \\
\hline 30 & Gajac & Gironde & 64 & Pernaut & Marne \\
\hline
\end{tabular}




\begin{tabular}{|c|c|c|c|c|c|}
\hline 65 & Ay & Marne & 97 & Dijon & Côte-d'Or \\
\hline 66 & Nancy & Meurthe-et- & 98 & Coëtquidan & Morbihan \\
\hline & & Moselle & 99 & Arouville & Meurthe-et- \\
\hline 67 & Rodez & Aveyron & & & Moselle \\
\hline 68 & Mourmelon & Marne & 100 & Brest & Finistère \\
\hline 69 & Mailly & Aube & 101 & Mâcon & Saône-et-Loire \\
\hline 70 & Saint-Lô & Manche & 102 & Vesoul & Haute-Saône \\
\hline 71 & Brinville & Calvados & 103 & La Prugue & Loire \\
\hline 72 & Cherbourg, & Manche & 104 & Hanches & Eure-et-Loire \\
\hline & & & 105 & Foix et & Ariège \\
\hline 73 & Cherbourg, & Manche & & Le Vernet & \\
\hline & camps $1,2,3$ & & 106 & Epernay & Marne \\
\hline 74 & Montpellier & Hérault & 107 & Tarbes & Hautes-Pyrénées \\
\hline 75 & Abbeville & Somme & 108 & Ger & Indre-et-Loire \\
\hline 76 & Dole & Jura & 109 & Saint-Paul & Lot \\
\hline 77 & Agen & Lot-et-Garonne & 110 & Libourne & Gironde \\
\hline 78 & Rivesaltes & $\begin{array}{l}\text { Pyrénées- } \\
\text { orientales }\end{array}$ & 111 & Salles & Bas-Rhin \\
\hline 9 & & Saône-et-Loire & 112 & Baillet-en-France & Seine-et-Oise \\
\hline 7 & Saône & SaOHe-et-LOHE & 113 & Sarreguemines & Moselle \\
\hline 80 & Saint-Pierre- & Manche & 114 & Suippes & Marne \\
\hline & l’Église & & 115 & Clermont- & Puy-de-Dôme \\
\hline 81 & Beaulieu & Gers & & Ferrand & \\
\hline 82 & Compiègne & Oise & 116 & Lugange & Meurthe-et- \\
\hline 83 & Soissons & Oise & & & Moselle \\
\hline 84 & Connantre & Marne & 117 & Bourges & Cher \\
\hline 85 & Châlons-sur- & Marne & 118 & Sens & Yonne \\
\hline & Marne & & 119 & Béthune & Pas-de-Calais \\
\hline 86 & Reims & Marne & 120 & Boulogne & Pas-de-Calais \\
\hline 87 & Sermaize-les- & Marne & 121 & Montreuil & Pas-de-Calais \\
\hline & Bains & & 122 & Morhange & Moselle \\
\hline 88 & Rambervillers & Vosges & 123 & Aynes & Puy-de-Dôme \\
\hline 89 & Arras & Pas-de-Calais & 124 & Verdun & Meuse \\
\hline 90 & Hénin-Liétard & Pas-de-Calais & 125 & Montestruc- & Gers \\
\hline 91 & Beauvais & Oise & & sur-Gers & \\
\hline 92 & Bordeaux & Gironde & 126 & Mutzig & Bas-Rhin \\
\hline 93 & Le Mans & Sarthe & 127 & Poitiers & Vienne \\
\hline 94 & Saint-Omer & Pas-de-Calais & 128 & Ronchin & Nord \\
\hline 95 & Mézières & Ardennes & 129 & Saint-Médard & Gironde \\
\hline 96 & Troyes & Aube & 130 & Viret & Ardennes \\
\hline
\end{tabular}

